Revue d'histoire de l'Amérique française

RAS REVUE D.HISTOIRE DE L'AMÉRIQUE FRANÇAISE

\title{
Les Acadiens dans une seconde patrie : La Louisiane (suite et fin)
}

\section{Jeanne Grégoire}

Volume 16, numéro 3, décembre 1962

URI : https://id.erudit.org/iderudit/302215ar

DOI : https://doi.org/10.7202/302215ar

Aller au sommaire du numéro

Éditeur(s)

Institut d'histoire de l'Amérique française

ISSN

0035-2357 (imprimé)

1492-1383 (numérique)

Découvrir la revue

Citer cet article

Grégoire, J. (1962). Les Acadiens dans une seconde patrie : La Louisiane (suite et fin). Revue d'histoire de l'Amérique française, 16(3), 428-435.

https://doi.org/10.7202/302215ar d'utilisation que vous pouvez consulter en ligne.

https://apropos.erudit.org/fr/usagers/politique-dutilisation/ 


\title{
LES ACADIENS DANS UNE SECONDE PATRIE : LA LOUISIANE *
}

\author{
(suite et fin)

\begin{abstract}
PAROISSE SAINT.GABRIEL, COTE D'IBERVILLE, LOUISIANE
\end{abstract}

BAPTEMES 14

Babin, Baptiste-Olivier, 24 avril 1773, 1er mars 1773, Olivier, Marguerite Brault.

Blanchard, Pierre-Isidore, 4 janv. 1773, 12 oct. 1772, Joseph, Marie-Joseph Landry.

Brault, Marie-Rose, 12 avril 1774, 17 janv. 1774, Patrice, Isabelle Richard.

Capdeville, Jean-Baptiste, 26 Xbris, 27 août 1773, Bernard, A Clouâtre.

Chiasson, M-Madeleine, 28 Xbris 1773, 12 Xbris, Paul, M-Madeleine Blanchard.

Clouâtre, Marine, 24 avril 1773, 26 oct. 1772, Patrice, Magdeleine Boudreau.

Dupuis, Jean, 20 avril 1773, 24 janv. 1773, Joseph, Anne Hébert.

Hamilton, Anne, 24 avril 1773, 19 mars 1773, Joseph, Aman Thesse.

Hébert, Anne-Marie, 21 oct. 1773, 20 oct. 1773, François, Marguerite Leblanc.

Hébert, Jean-Marie, 1773, 30 août 1773, Patrice, Marguerite Leblanc.

Hébert, Marie-Geneviève, 21 Xbris 1773, 20 xbris, Jean-Baptiste, Marguerite Dupuis.

Hébert, Thomas, -, 18 janv. 1773, Aman, Marie Landry.

Landry, Ignace, 24 avril 1773, 1er fév. 1773, François, Marguerite Leblanc.

Landry, Joseph Deneau, 22 avril 1773, 14 mars 1773, Joseph, Marguerite Leblanc.

Landry, Joseph-Eusèbe, 10 avril 1773, 18 mars 1773, AnselmeAlexandre, Osite Landry.

* Voir notre Revue, XV: 572-593; XVI: 105-116, 254-266.

14 Cette liste de baptêmes est donnée dans l'ordre suivant: nom, date de baptême, date de naissance, père, mère. 
Landry, Marie, 24 avril 1773, 15 mars 1773, Hyacinthe, Marguerite Landry.

Melançon, Louis-Grégoire, 24 avril 1773, 21 sept. 1772, Aman, Amaque Babin.

Polome, Marguerite, 22 avril 1773, 13 mars 1773, Félix-Joseph, Marguerite Leblanc.

Richard, Pierre-Grégoire, 11 avril 1774, 17 janv. 1774, Mathurin, Elisabeth-Mad. Landry.

Richard, Simon, 28 avril 1773, 25 mars 1773, Simon, Marie-Rose Landry.

\section{MARIAGES}

Arbour, François, f. de François (déf.) de Bâton Rouge et de Marie Henry, m. le 27 sept. 1790, avec Marie-Jeanne Daigle, f. de déf. François et de Jeanne Hollet, de Manchac.

Aucoin, François, f. de Pierre et de Marie Guédry, m. le 19 janv. 1818, avec Marie-Rose Richard, veuve de Bernard Comeaux, f. de Joseph et de Cécile Dupuis.

Aucoin, Mathilde, f. min. de feu Pierre et d'Elizabeth Garenil, m. le 15 janv. 1846, avec Lenfroy Hébert, f. de feu Joseph et de L. Verdais.

Aucoin, G ou Trasimond, f. de feu François et de Marie-Rose Richard, m. le 24 oct. 1844, avec Solidaine LeBlanc, f. de feu Maximilien et d'Hélène Alain.

m. 2o, le 10 avril 1856, avec Marie-B. Beronne, native de Savoie, f. de Pierre et de Marie Ambloy.

Babin, François, f. de Simon et d'Anastasie Tériot, m. le 31 déc. 1786, avec Marie Vuet, f. d'Ignace et de Cécile Tulk, natifs de Saint-Malo.

Babin, Paul, f. de Dominique et de Marguerite Boudreau, m. le 24 fév. 1784, avec Marguerite Brasseur, f. de Pierre et d'Elizabeth Richard.

Beaudin, Jean-Louis, f. de Pierre et d'Anne Messonet, natifs de l'île Noirmoutier, France, m. le 28 janv. 1787, avec Françoise Doiron, f. de Grégoire et d'Hélène Aucoin, natifs de SaintMalo.

Bergeron, Estevan (Stephens ?), f. de Guillaume et d'Agnès Lardover, m. le 15 sept. 1781, avec Françoise Olend, f. de Pierre, habitant des Arcas.

Blanchard, Antoine, f. d'Anselme et d'Esther LeBlanc, natifs du Canada, m. le 22 avril 1788, avec Marie-Joseph Hébert, native de cette colonie, f. de Joseph et de Marie Landry, natifs du Canada. 
Blanchard, Jérôme, f. d'Anselme et d'Esther LeBlanc, m. le 11 fév. 1787, avec Marine Clouâtre, f. de Pierre et de Madeleine Boudreau.

Blèque, Guillaume, $f$. de Jean et de Marguerite O'Donnel, de S.Jean de Mouster, en Irlande, m. le 17 sept. 1793, avec Marguerite Arbour, f. d'Antoine et de Cécile Doiron.

Boudreau, Francois-Xavier, veuf de Marguerite Dugas, f. d'Antoine et de Brigitte Grante, m. le 23 mai 1787, avec MarieFrançoise LeBlanc, f. de Joseph et d'Anne Hebert.

Boudreau, Jean-Baptiste, veuf de M-Modeste Trahan, f. d'Alexandre et de Marie-Madeleine Verreaut, de l'Assomption, Canada, m. le 26 fév. 1786, avec Anna Joseph Hanire (?), veuve de Théodore Thériot, $f$. de Pierre et de Madeleine Pitret, de l'Assomption.

Brault, Joseph, f. de Joseph et de Joséphine Landry, m. le 4 juillet 1786, avec Hélène Hamilton, f. de Joseph et d'Anastasie Comme.

Bruneteau, Pierre, f. de Jean et d'Anne Bosüne, natifs de Hala, en Champagne, m. le 5 mars 1785, avec Anne Hébert, veuve de Joseph Dupuy.

Charpet, Joseph, f. de Paul et d'Isabelle Sarlet, natifs de Maxilen, pays américain, m. le 26 oct. 1785 , avec Mariane Choquet, $f$. de Joseph et de Mariane Decous, de la Pointe Coupée.

Clouâtre, Joseph, f. de Pierre et de Marguerite LeBlanc, m. le 13 avril 1787, avec Isabelle Thibodeau, f. d'Olivier et d'Isabelle Boudreau.

Clouâtre, Joseph, f. de Pierre et de Marguerite LeBlanc, m. le 3 juin 1785, avec Marguerite Babin, f. de Dominique et de Marguerite Boudreau.

Comeau, Anne, f. de Jean et d'Elizabeth Hébert, acadiens, m. le 11 janv. 1773, avec Jean-Baptiste Doucet, f. de Charles et de Madeleine Landry. (dispense de 3 bans).

Comeau, Estelvan (Stephen ?), f. d'Alexandre et de Marguerite Babin, m. le 30 mai 1792, avec Marguerite Blanchard, veuve, f. de Joseph et de Marie-Jeanne Landry.

Comeau, Jean-Charles, veuf, $f$. de Charles et de Marie-Madeleine Landry, m. le 9 juillet 1781, avec Catalina Buch, convertie, f. de Daniel et de Daina Luisse, protestants, natifs de la Virginie.

Comeau, Pierre, f. d'Alexis et de Marguerite Babin, m. le 10 janv. 1785, avec Claire Brault, f. de Joseph et de Marie Landry.

Culère, Jean-Pierre, de Nantes, Bretagne, f. de Jean et de Françoise de La Mothe, $\mathrm{m}$. le 16 nov. 1790, avec Madeleine Le 
Blanc, veuve d'Estevan Babin, f. de Venture et de Marie Thériault, de Baltimore.

Daigle, Charles, veuf de Anne Vinsaut, m. le 5 fév. 1786, avec Marie Boudreau, veuve de Maxime Dugas (tous natifs de la paroisse Ste-Famille, dans l'Acadie).

Dègre, Paul Olivier, natif de France, f. d'Olivier et de Marie Melançon, m. le 8 sept. 1788, avec Marie-Jeanne Prichal, f. de Pierre et de Françoise Dègre.

Deglet, Victor, f. d'Olivier et de Blanche LeBlanc, natifs d'Angleterre, m. le 8 mai 1786, avec Marguerite Doiron, f. de Joseph et d'Anne Thibodeau, natifs de Saint-Malo.

Dominguez, Pedro, f. de Sébastien et de Lorenza Martinez, de Ste-Anne de Orcana de Séville en Espagne, m. le 10 juillet 1786, avec Jeanne Cabon, f. de Jean et de Françoise Damon, de France, veuve de Pierre Dugas.

Dupuis, Joseph, f. de Joseph et d'Anne Hébert, m. le 15 avril 1792, avec Marie de Viller, f. de Balthasar et de MarieFrançoise Boisir.

Forest, Moise, f. de Jean-Baptiste et de Marguerite Bibiane Hébert, m. le 12 avril 1790, avec Marguerite Bibiane Alain, f. de Pierre et de Catherine Hébert.

Gale, Abner, f. de Moise et de Marie Caltarvat, de Boston, m. le 5 mars 1795, avec Anne Adélaide Hébert, f. d'Etienne et de Marie Landry.

Gareuille, Michel, natif de Marseille, f. d'André et d'Isabelle Mercuron, m. le 21 mai 1793, avec Rosalie LeBlanc, f. de Joseph et de Marguerite Landry.

Guédry, Firmin, f. de Jean-Baptiste et d'Anne Dupuis, paroisse de l'Assomption, m. le 19 fév. 1786, avec Marguerite Landry, f. de Charles et de Marguerite Boudreau, originaire de Saint-Servan, diocèse de Saint-Malo.

Guédry, Jean-Baptiste, f. de Pierre et de Marcellin Laforest, de l'Ascension, m. le 14 sept. 1840, avec Joséphine Gaudin, f. de François Desalles et de Delphine Landry.

Hamilton, Ignace, f. de Joseph et d'Anastasie Comeau, du Maryland, m. le 3 nov. 1790, avec Anne Catalina Bouche, f. de Daniel et de Jeanne Louise de Philadelphie.

Hébert, Alexandre, f. de François et d'Itha Nauran, m. le 26 janv. 1789 , avec Marie-Jeanne Thibodeau, f. de Joseph et de Louise Viachée.

Hébert, Benoni, f. de François et de Marie LeBlanc, m. le 7 déc. 1793, avec Madeleine Alain, f. de Pierre et de Catherine Hébert (dispense de parenté). 
Hébert, Charles, veuf, f. de Pierre et de Marguerite LeBlanc, m. le 17 juillet 1792, avec Anne Godreau, veuve, f. d'Aman et de Marguerite Landry.

Hébert, Charles, f. de Pierre et de Marguerite LeBlanc, m. le 18 mai 1785, avec Madeleine Brault, f. de Janvier et de Osite Landry.

Hébert, Ignace, veuf de Marie LeBlanc, m. le 11 janv. 1773, avec Rosalie Babin, veuve de Joseph Babin.

Hébert, Ignace, f. de Paul et de Marguerite Melançon, m. le 26 nov. 1781, avec Marie Madeleine Beauvais, veuve de Pierre Landry, f. de Bonaventure et de Clara Rivest.

Hébert, Jean-Baptiste, f. de François et de Marie LeBlanc, m. le 18 mai 1785 , avec Marine Hébert.

Hense, Charles, f. d'Ignace et de Cécile Bourque, natifs de S-Servan, France, m. le 13 nov. 1786, avec Marie Aché, f. d'Antoine et de Marie Clemenson, de S-Servan.

Hermandez, Olivier, f. de Diego et de Théotiste Babin, m. le 19 mai 1792, avec Marine Hébert, f. de Marin et de Marie-Claire Landry.

Horrion, Joseph, f. de Joseph et de Rosa Breault, m. le 1er mars 1794, avec Clothilde Marion, f. de François et de Jeanne Guio.

Lambremont, Michel, f. de Pierre et de Françoise Clois, de la Nouvelle-Orléans, m. le 15 oct. 1793, avec Marguerite Brault, veuve de Pierre LeBlanc, f. de Jean et de Marguerite Landry.

Landry, Alexandre, f. de Joseph et de Marie Richard, natifs de l'Acadie, m. le 6 fév. 1786, avec Marie-Modeste Hébert, f. d'Aman et de Marie Richard, natifs de Morlais.

Landry, Augustin, veuf de Marie Forest, f. de Joseph et de Marie Nixain, de l'Acadie, m. le 5 août 1786, avec Isabelle Landry, veuve d'Estevan Rivest, $f$. de Pierre et de Clara Babeu.

Landry, Firmin, veuf, f. d'Alexandre et de Anna ?, m. le 6 fév. 1792, avec Marie Hamilton, veuve, f. de Joseph et d'Anastasie Comeau.

Landry, Firmin-Pancrace, f. de Charles et de Marie-Anne Boudreau, de S-Malo, m. le 18 oct. 1790, avec Mariane Babin, f. de Firmin et de Bibiane Brault.

Landry, Jean-Baptiste, f. d'Estevan et de Marie-Joseph Landry, de la Fourche, m. le 23 nov. 1789, avec Marie-Madeleine Hébert, f. de Jean et de Marie Dupuis.

Landry, Jean-Baptiste, f. de Pierre et de Geneviève Bruchar, m. le 26 nov. 1786, avec Marie LeBlanc, f. de Jean-Charles et de ? Landry.

Landry, Joseph, f. d'Augustin et de Marie Babin, m. le 8 oct. 1787, avec Oliva Isabelle Brault, f. d'Honoré et d'Isabelle LeBlanc. 
Landry, Pierre, f. de Pierre et de Marie Landry, m. le 20 nov. 1786, avec Rosalie Hébert, f. de François et de Marie LeBlanc.

Landry, Pierre, f. d'Abraham et de Marguerite Hanc, paroisse de l'Ascension, m. le 11 janv. 1773, avec Marguerite Alain, f. de Pierre et de Catherine Hébert.

Landry, Pierre-Joseph, f. de Pierre et de Marie-Joseph Hébert, défunts, m. le 30 juin 1790, avec Scholastique Brault, f. de déf. Joseph et de Marie-Josephte Landry.

Landry, Simon, f. de ? et de Marie Hébert, m. le 9 déc. 1833, avec Félicité Arsenaux, f. d'Abraham et de Marie-Eloise LeBlanc.

Landry, Jean, natif du Canada, f. de Jean et de Geneviève Brucchal, m. le 23 oct. 1788, avec Marie-Itha Blanchard, native de cette paroisse, $f$. de Joseph et de Josephte Landry.

Landry, Jean-Baptiste, natif du Canada, f. de Pierre et de Geneviève Buchal, m. le 18 août 1788, avec Anne-Marie Blanchard, native de cette paroisse, f. de déf. Joseph et de Marie-Joseph Landry.

Lavergne, Valsin, f. de feu Louis et de feu Lucie Brault, m. le 17 juin 1773, avec Marie-Elmire Gaudin, f. d'Edouard et de feu Madeleine Landry.

LeBlanc, Isaac, f. de Bonaventure et de Marie Thériault, m. le 13 nov. 1786, avec Félicité Melançon, f. d'Aman et d'Anne Babin.

LeBlanc, Jean-Baptiste, f. de Jean-Charles et de Judith Landry, m. le 22 avril 1781, avec Madeleine-Marthe Forest, f. de Jean-Baptiste et de Marguerite Richard.

LeBlanc, Joseph, f. de Michel et de Marie-Josephte Trahan, m. le 18 juin 1781, avec Marguerite Landry, f. d'Augustin et de Marie Babin.

LeBlanc, Joseph, f. de Simon et de Marie Trahan, natifs de SaintMartin, France, m. le 7 fév. 1787, avec Marguerite LeBlanc, f. de Joseph et d'Anne Hébert, natifs de Plausen, France.

Lejeune, Servan Mathurin, f. d'Anastase et de Marie Caret, m. le 11 janv. 1792, avec Marguerite Pierre, f. de déf. Simon et de Geneviève Richard.

Martinez, Joseph, f. de Balthazar et de Rosa Yacon, de Ste-Croix, Iles Canaries, m. le 19 mai 1794, avec Marine Landry, f. de Pierre et d'Anne Thériault, de S-Malo.

Mathé, Simon, de S-Estevan de Moran, diocèse de La Rochelle, f. de Siméon et de Marie-Madeleine Toutant, m. le 1er déc. 1792, avec Marie Rivas, f. de François et de Lucia Métrinque.

Melançon, Grégoire, f. d'Aman et d'Anne Babin, m. le 21 avril 1792, avec Christine Landry, f. d'Augustin et de Marie Force.

Mire, Joseph, f. de Pierre et d'Isabelle Thibodeau, de l'Acadie, m. le 22 mai 1786, avec Marie-Marguerite Daigre, f. de 
Simon-Pierre et de Marie-Madeleine Thériault, natifs d'Angleterre.

Morales, Jean, f. de Jean et de Marie-Louise Castillo, de Séville, Espagne, m. le 30 juin 1792, avec Marine Clouâtre, f. de Georges et de Marguerite Landry.

Oguan, Pierre, du Canada, f. de Charles et de Madeleine Trahan, défunts, m. le 24 oct. 1788, avec Marie Landry, native du Canada, f. de G. et d'Anne Moly.

Park, Olivier, f. de Pierre et d'Angélica Gaudin, natifs de l'Acadie, m. le 15 janv. 1787, avec Marie Dupuis, f. de JeanBaptiste et de Anne Richard.

Perrin, Edmée-Elizabeth, f. de Nicolas et d'Anne Buguen, natifs de Nogent-sur-Seine, Champagne, m. le 9 juin 1786, avec Antonia Suarez, f. de Pierre Pedro Allemand, et de Marie Agular, natifs de la ville d'Aguines, Iles Canaries.

Prosper, Jean, f. de Jean et de Marie Hus, natifs de Languedoc, b. à Carcassonne, m.: le 17 juillet 1787, avec Marguerite Lavergne, f. de Pierre et de Marguerite Daigle, de Guabre de Gras, en Normandie.

Provencher, Daniel, f. de Daniel et de Thérèse Lacroix, m. le 11 sept. 1792, avec Marie Daigle, f. de Simon-Pierre et de Madeleine Thériault.

Rivest, Blaise, f. de Michel et d'Anne Landry, natifs de l'Acadie, m. le 1er avril 1788, avec Marie-Marguerite Noel, f. de Pierre et de Marie-Marguerite Barbe, natifs d'Angleterre, Europe.

Rivest, Théodore, f. d'Etienne et de Claire Forest, m. le 1er déc. 1779, avec Esther LeBlanc, f. de Bonaventure et de Marie Thériault.

Robichaud, Charles, f. de Charles et de Marguerite Le Blanc, $\mathrm{m}$. le 19 fév. 1792, avec Jeanne-Marie Henry, f. de Charles et de Marguerite Thériault, anciens habitants de Bâton Rouge.

Robichaud, François-Xavier, f. de Joseph et d'Anne Hébert, natifs de Saint-Malo, m. le 27 juillet 1790, avec Marguerite Landry, f. d'Athanase et de Madeleine Hébert.

Roth, Philippe, f. de Joseph et d'Antoinette Lamarre, m. le 8 fév. 1791, avec Marie-Joseph Horrion, f. de Joseph et de Rosa Brault.

Savoy, Pierre, f. de Simonet et de Rosalie Dubon, paroisse de l'Ascension, m. le 12 fév. 1840, avec Marie-Adèle Ory, f. de feu Nicolas et d'Adéline Bourg.

Templet, Jean, f. d'André et de Marguerite LeBlanc, natifs de Saint-Servan, diocèse de S-Malo, $m$. le 5 oct. 1785, avec Mariane Doiron, f. d'Alexandre et d'Ursule Hébert, paroisse de Plailain, diocèse de Saint-Malo. 
Trahan, Jean-Baptiste, f. de Pierre-Simon et de Joséphine Granger, m. le 22 mai 1786, avec Anne-Geneviève Daigre, f. de Simon-Pierre et de Marie-Madeleine Thériault, natifs d'Angleterre.

Trahan, Jean-Paul, f. de Jean et de Marguerite Clément, natifs de S-Malo, m. le 30 mars 1788, avec Marie-Joseph Va Toune, f. de Grégoire et d'Hélène de Monde, natifs de l'Acadie.

Tullié, Isidore, f. d'Ernest (déf.) et de Colette Arnault, de Manchac, m. le 27 sept. 1790, avec Marie-Louise Daigle, f. de déf. François et de Jeanne Nollet.

Turenne, Pierre-Paul, f. de déf. Charles et de Cécile Béraud, originaire de Bretagne, b. à Plaidel, m. le 4 janv. 1788, avec Marguerite Segolut, f. d'Emilien et de Marguerite Moquin, originaires de Vannes, en Bretagne.

Usé, Jean-Baptiste, f. d'Ignace et de Cécile Bourque, $\mathrm{m}$. le 10 nov. 1791, avec Françoise Henri, f. de Charles et de Françoise Hébert.

NOTE: Les autres actes de mariages portent des noms étrangers, originaires de Santa Cruz, d'Allemagne, de Cuba, des Guyanes, etc. Le même registre contient aussi les actes de baptême qui suivent:

Il est souvent fait mention dans les actes de mariage de la paroisse Saint-Gabriel et Saint-Bernard, Côte de Manchac.

JEANNE GRÉGOIRE 